\title{
ON STRUCTURAL COMPLETENESS VS ALMOST STRUCTURAL COMPLETENESS PROBLEM: A DISCRIMINATOR VARIETIES CASE STUDY
}

\author{
MIGUEL CAMPERCHOLI, MICHAE M. STRONKOWSKI, AND DIEGO VAGGIONE
}

\begin{abstract}
We study the following problem: Determine which almost structurally complete quasivarieties are structurally complete. We propose a general solution to this problem and then a solution in the semisimple case. As a consequence, we obtain a characterization of structurally complete discriminator varieties.

An interesting corollary in logic follows: Let $L$ be a propositional logic/deductive system in the language with formulas for verum, which is a theorem, and falsum, which is not a theorem. Assume also that $L$ has an adequate semantics given by a discriminator variety. Then $L$ is structurally complete if and only if it is maximal. All such logics/deductive systems are almost structurally complete.
\end{abstract}

\section{INTRODUCTION}

A quasivariety $\mathcal{Q}$ is structurally complete ( $\mathrm{SC}$ for short) if all its admissible (true in free algebras) quasi-identities are true in $\mathcal{Q}$ [1]. And $\mathcal{Q}$ is almost structurally complete (ASC for short) if every one of its admissible quasi-identities which do not hold in $\mathcal{Q}$ is passive $[13,27]$. These are quasi-identities whose premises are not satisfiable in free algebras. Both notions have their origins in the theory of deductive systems. The SC property was introduced by Pogorzelski in [30] and the ASC property by Dzik in [11]. From this perspective both of them serve the same purpose: to separate deductive systems whose "deductive power" cannot be strenghtened by adding new rules while keeping the set of theorems unchanged. Indeed, such strenghtening cannot be obtained by adding passive rules. A detailed discussion concerning the logical context may be found in [13].

There are important deductive systems, like S5 modal logic augmented by the modus ponens rule and the necessitation rule, or $€$ ukasiewicz $n$-valued logic augmented by the modus ponens rule, which are not SC merely because of the underivability of some passive rules. Thus they are ASC. (Note that both these examples have adequate semantics given by discriminator varieties.) One then can ask: are

2010 Mathematics Subject Classification. 08C15,03G25,03B22,08B20.

Key words and phrases. Structural completeness, almost structural completeness, discriminator varieties, semisimple quasivarieties, minimal varieties, minimal quasivarieties.

The work of the first and third authors was supported by Conicet and Secyt-UNC. The work of the second author was supported by the Polish National Science Centre [DEC2011/01/D/ST1/06136]. 
such examples rare, or maybe there are plenty of them (in whatever sense)? This leads us to the following task.

SC vs ASC problem. Determine which ASC qausivarieties/deductive systems are SC.

There are two, quite obvious, conditions for an ASC quasivariety to be SC: minimality and the existence an idempotent element in a free algebra. A relaxation of the second condition would be that every nontrivial algebra from the quasivariety under consideration admits a homomorphism into a free algebra. And indeed, it was shown in [13, Corollary 3.5.] that it yields SC for ASC quasivarieties. Still, there are SC quasivarieties in which this condition fails, see Examples 5.10 and 5.11. Nonetheless, our first result in this paper says that it is quite close to the right condition. We prove that an ASC quasivariety $\mathcal{Q}$ is $\mathrm{SC}$ iff every nontrivial algebra in $\mathcal{Q}$ admits a homomorphism into some elementary extension of a free algebra for $\mathcal{Q}$ iff every nontrivial $\mathcal{Q}$-finitely presented algebra admits a homomorphism into a free algebra for $\mathcal{Q}$ (Theorem 3.3).

In the subsequent considerations we restrict to semisimple quasivarieties. We prove that an ASC semisimple quasivariety is SC iff it is minimal or there exists an idempotent element in some elementary extension of a free algebra (Theorem 4.2). This, in particular, yields a characterization of SC discriminator varieties (Theorem 5.5). Indeed, discriminator varieties are semisimple [8, Theorem IV.9.4]. Moreover, Burris proved in [7, Theorem 3.1] that every discriminator variety has projective unification (see also [34]). This property is known to imply ASC [12, Corollary 6][13, Corollary 5.1]. Discriminator varieties, introduced by Pixley in [29], form a restrictive class from the perspective of universal algebra. On the other hand, algebras in discriminator varieties have a well understood structure as Boolean products of simple and trivial algebras $[6,8,33]$. (We used it to show that minimal discriminator varieties are also minimal as quasivarieties, see Proposition 5.4). Moreover, discriminator varieties appear quite often in algebraic logic, see e.g. $[14,16,17,19,22,23,24,25]$. In most of these cases there are distinct constants for verum and falsum. For such varieties, being SC is equivalent to being minimal (Corollary 5.6).

We would like to emphasize that in our main result we do not impose any finiteness condition, like local finiteness or the finiteness of the language. Note that such conditions where commonly used in this area (cf. e.g. [1]).

It is worth adding that almost all (in a certain strict sense) finite algebras in a finite language with at least one at least binary operation generate minimal discriminator variety. They are also minimal as quasivarieties and hence are SC. It was proved by Murskii in [28] (see also [2, Chapter 6]) that almost all finite algebras with at least one at least binary operation have all their idempotent operations as term operations (they are idemprimal). In particular, they have the discriminator operation as a term operation and do not have proper subalgebras.

Finally note that the SC vs ASC problem was solved in [13, Proposition 8.6] for varieties of closure algebras (equivalently for normal extensions of S4 modal logic) 
and for varieties of bounded lattices [13, Proposition 7.7]. Both of these cases are quite different than the case of discriminator varieties. It is shown there that an ASC variety of closure algebras is $\mathrm{SC}$ iff it does not contain a four-element simple algebra iff it satisfies the McKinsey identity. Note that a nontrivial closure algebra satisfies the McKinsey identity iff it admits a homomorphism onto a two-element algebra (a two-element closure algebra is free in every nontrivial variety of closure algebras). And a variety of bounded lattices is SC iff it is ASC iff it satisfies distributivity law.

\section{Preliminaries}

In the paper we assume that the reader has basic knowledge in universal algebra $[8,2]$ and model theory $[10,18]$. However, we recall needed concepts from quasivariety theory $[15,26]$ since it is less known.

By a quasi-identity we mean a sentence in a first order algebraic language of the form

$$
(\forall \bar{x})\left[s_{1}(\bar{x}) \approx t_{1}(\bar{x}) \wedge \cdots \wedge s_{n}(\bar{x}) \approx t_{n}(\bar{x}) \rightarrow s(\bar{x}) \approx t(\bar{x})\right],
$$

where $n \in \mathbb{N}$. We allow $n$ to be zero, and in such case we call the sentence an identity. It will be convenient to have a more compact notation for quasi-identities, and we will often write them in the form

$$
(\forall \bar{x})[\varphi(\bar{x}) \rightarrow \psi(\bar{x})],
$$

where $\varphi$ is a conjunction of equations (i.e., atomic formulas) and $\psi$ is an equation. We call $\varphi$ the premise and $\psi$ the conclusion of a quasi-identity.

By the (quasi-)equational theory of a class $\mathcal{K}$ of algebras in the same language we mean the set of (quasi-)identities true in $\mathcal{K}$. A (quasi)variety is a class defined by (quasi-)identities. Equivalently, a class of algebras is a quasivariety if it is closed under taking substructures, direct products and ultraproducts. If it is additionally closed under taking homomorphic images, it is a variety. (We tacitly assume that all considered classes contain algebras in the same language and are closed under taking isomorphic images. Also all considered class operators are assumed to be composed with the isomorphic image class operator.) A (quasi)variety is trivial if it consists of one-element algebras, and is minimal if it properly contains only the trivial (quasi)variety. We say that a (quasi)variety $\mathcal{Q}$ is generated by a class $\mathcal{K}$ if it is the smallest (quasi)variety containing $\mathcal{K}$. This means that $\mathcal{Q}$ is defined by the (quasi-)equational theory of $\mathcal{K}$. We denote such a (quasi)variety by $\mathrm{V}(\mathcal{K})(\mathrm{Q}(\mathcal{K})$ respectively). When $\mathcal{K}=\{\mathbf{A}\}$ we simplify the notation by writing $\mathrm{V}(\mathbf{A})(\mathrm{Q}(\mathbf{A})$ respectively). Let $\mathrm{S}, \mathrm{P}, \mathrm{P}_{\mathrm{U}}, \mathrm{E}_{\mathrm{el}}$ be the subalgebra, direct product, ultraproduct and elementary extension class operators.

Proposition 2.1 ([8, Theorem V.2.25][15, Corollary 2.3.4]). For a class $\mathcal{K}$ of algebras in the same language we have $\mathrm{Q}(\mathcal{K})=\mathrm{SPP}_{\mathrm{U}}(\mathcal{K})$. In particular, for an algebra $\mathbf{A}$ we have $\mathrm{Q}(\mathbf{A})=\mathrm{SPE}_{\mathrm{el}}(\mathbf{A})$.

Let $\mathcal{Q}$ be a quasivariety. A congruence $\alpha$ on an algebra $\mathbf{A}$ is called a $\mathcal{Q}$-congruence provided $\mathbf{A} / \alpha \in \mathcal{Q}$. Note that $\mathbf{A} \in \mathcal{Q}$ if and only if the equality relation on $A$ is a 
$\mathcal{Q}$-congruence of $\mathbf{A}$. The set $\operatorname{Con}_{\mathcal{Q}}(\mathbf{A})$ of all $\mathcal{Q}$-congruences of $\mathbf{A}$ forms an algebraic lattice. It is a meet-subsemilattice of $\operatorname{Con}(\mathbf{A})$, the lattice of all congruences of $\mathbf{A}$ [15, Corollary 1.4.11].

A nontrivial algebra $\mathbf{S}$ is $\mathcal{Q}$-simple if $\mathrm{Con}_{\mathcal{Q}}(\mathbf{S})$ has exactly two elements: the equality relation $\operatorname{id}_{S}$ on $S$ and the total relation $S^{2}$ on $S$. A nontrivial algebra $\mathbf{S} \in \mathcal{Q}$ is $\mathcal{Q}$-subdirectly irreducible if the equality relation on $A$ is completely meet irreducible in $\operatorname{Con}_{\mathcal{Q}}(\mathbf{A})$. (In the case when $\mathcal{Q}$ is a variety we drop the prefix " $\mathcal{Q}-$-") The importance of $\mathcal{Q}$-subdirectly irreducible algebras follows from the fact that they determine $\mathcal{Q}$. Indeed, in an algebraic lattice each element is a meet of completely meet-irreducible elements. Thus we have the following fact.

Proposition 2.2 ([15, Theorem 3.1.1]). Every algebra in $\mathcal{Q}$ is isomorphic to a subdirect product of $\mathcal{Q}$-subdirectly irreducible algebras. In particular, the class of $\mathcal{Q}$-subdirectly irreducible algebras generates $\mathcal{Q}$.

A quasivariety $\mathcal{Q}$ is semisimple if every $\mathcal{Q}$-subdirectly irreducible algebra is $\mathcal{Q}$ simple. So if $\mathcal{Q}$ is semisimple, then it is generated by $\mathcal{Q}$-simple algebras.

Let $\mathbf{G} \in \mathcal{Q}$ and $W \subseteq G$. We say that $\mathbf{G}$ is free for $\mathcal{Q}$ over $W$, and is of rank $|W|$, if $\mathbf{G} \in \mathcal{Q}$ and it satisfies the following universal mapping property: every mapping $f: W \rightarrow A$, where $A$ is a carrier of an algebra $\mathbf{A}$ in $\mathcal{Q}$, is uniquely extendable to a homomorphism $\bar{f}: \mathbf{G} \rightarrow \mathbf{A}$. In our considerations the following weaker property will be used. If $\mathbf{G}$ is free for $\mathcal{Q}$ and $\mathbf{A} \in \mathcal{Q}$, then there is a homomorphism from $\mathbf{G}$ into $\mathbf{A}$ (we adopt the convention that algebras have nonempty carriers). If $\mathcal{Q}$ contains a nontrivial algebra, then it has free algebras over arbitrary non-empty sets and, in fact, they coincide with free algebras for the variety $\mathrm{V}(\mathcal{Q})$. (Note here that $\mathrm{V}(\mathcal{Q})$ is the class of all homomorphic images of algebras from $\mathcal{Q}$.) Let us fix a denumerable set of variables $V=\left\{v_{0}, v_{1}, v_{2}, \ldots\right\}$. We denote a free algebra for $\mathcal{Q}$ over $V$ by $\mathbf{F}$ and the free algebra for $\mathcal{Q}$ over $V_{k}=\left\{v_{0}, v_{1}, \ldots, v_{k-1}\right\}$ by $\mathbf{F}(k)$. One may construct $\mathbf{F}$ and $\mathbf{F}(k)$ by forming the algebra of terms over $V$, or $V_{k}$ respectively, and taking a quotient with respect to the congruence that identifies terms $s(\bar{v}), t(\bar{v})$ determining the same term operation on every algebra from $\mathcal{Q}$ (in other words, when $\mathcal{Q}=(\forall \bar{x})[t(\bar{x}) \approx s(\bar{x})])$. We will notationally identify terms with the elements of $\mathbf{F}$ that they represent.

For an algebra $\mathbf{A}$ and a set $H \subseteq A^{2}$ there exists the least $\mathcal{Q}$-congruence $\theta_{\mathcal{Q}}(H)$ on A containing $H$. We say that an algebra is $\mathcal{Q}$-finitely presented if it is isomorphic to an algebra of the form $\mathbf{F}(k) / \theta_{\mathcal{Q}}(H)$ for some natural number $k$ and some finite set $H$ [15, Chapter 2]. For a tuple $\bar{x}=\left(x_{0}, \ldots, x_{k-1}\right)$ of variables and a conjunction of equations $\varphi(\bar{x})=s_{1}(\bar{x}) \approx t_{1}(\bar{x}) \wedge \cdots \wedge s_{n}(\bar{x}) \approx t_{n}(\bar{x})$ let

$$
\mathbf{P}_{\varphi(\bar{x})}=\mathbf{F}(k) / \theta_{\mathcal{Q}}\left(\left\{\left(s_{1}(\bar{v}), t_{1}(\bar{v})\right), \ldots,\left(s_{n}(\bar{v}), t_{n}(\bar{v})\right)\right\}\right),
$$

where $\bar{v}=\left(v_{0}, \ldots, v_{k-1}\right)$. Note that every finitely presented algebra is isomorphic to some $\mathbf{P}_{\varphi(\bar{x})}$. In the following fact we notationally identify variables from $\bar{v}$ with their congruence classes.

Fact 2.3. 
(1) A quasivariety $\mathcal{Q}$ satisfies a quasi-identity $(\forall \bar{x})[\varphi(\bar{x}) \rightarrow \psi(\bar{x})]$ if and only if $\mathbf{P}_{\varphi(\bar{x})} \models \psi(\bar{v})$.

(2) Given a conjunction of equations $\varphi(\bar{x})$ and an algebra $\mathbf{A} \in \mathcal{Q}$, there exists a homomorphism from $\mathbf{P}_{\varphi(\bar{x})}$ into $\mathbf{A}$ if and only if $\mathbf{A} \models(\exists \bar{x}) \varphi(\bar{x})$.

\section{General solution}

Let $\mathcal{Q}$ be a quasivariety and $\mathbf{F}$ be the free algebra of denumerable rank for $\mathcal{Q}$. A quasi-identity which is true in $\mathbf{F}$ is called $\mathcal{Q}$-admissible. For a quasi-identity $q=(\forall \bar{x})[\varphi(\bar{x}) \rightarrow \psi(\bar{x})]$ let

$$
q^{*}=(\forall \bar{x})[\neg \varphi(\bar{x})] .
$$

We say that a $\mathcal{Q}$-admissible quasi-identity $q$ is $\mathcal{Q}$-passive if $\mathbf{F}=q^{*}$, and $\mathcal{Q}$-active otherwise. By Fact 2.3, a $\mathcal{Q}$-admissible quasi-identity $q$ is $\mathcal{Q}$-active iff $\mathbf{P}_{\varphi(\bar{x})}$ admits a homomorphism into $\mathbf{F}$, where $\varphi(\bar{x})$ is the premise of $q$.

Example 3.1. in order to gain some intuition, let us consider one example. Let $\mathcal{R}$ be the variety of unitary rings and define the following quasi-identites in the language of $\mathcal{R}$ :

$$
\begin{aligned}
& q_{0}=(\forall x)[x+x \approx 0 \rightarrow x \approx 0], \\
& q_{1}=(\forall x)[x+x \approx 0 \wedge x \approx 1 \rightarrow x \approx 0] .
\end{aligned}
$$

Since they do not hold in the ring $\mathbb{Z}_{2}$ of integers modulo 2 , they do not hold in $\mathcal{R}$. But they are $\mathcal{R}$-admissible. Recall that the ring $\mathbb{Z}[V]$ of polynomials with noncommuting indeterminates from $V$ and integer coefficients is free for $\mathcal{R}$ over $V$. We have $\mathbb{Z}[V] \models q_{0}$ and $\mathbb{Z}[V] \models q_{1}$. Moreover, $q_{0}$ is $\mathcal{R}$-active, since $0+0=0$ in $\mathbb{Z}[V]$, and $q_{1}$ is $\mathcal{R}$-passive, since $0 \neq 1$ in $\mathbb{Z}[V]$. Note also that $\mathbf{P}_{x+x \approx 0}$ is isomorphic to $\mathbb{Z}\left[v_{0}\right] /\left(v_{0}+v_{0}\right)$ and admits a homomorphism onto $\mathbb{Z}$. The $\operatorname{ring} \mathbf{P}_{x+x \approx 0 \wedge x \approx 1}$ is isomorphic to $\mathbb{Z}_{2}$ and does not admit a homomorphism into $\mathbb{Z}$.

A quasivariety $\mathcal{Q}$ is structurally complete $(\mathrm{SC})$ provided that every $\mathcal{Q}$-admissible quasi-identity is true in $\mathcal{Q}$. In other words, if $\mathcal{Q}=\mathrm{Q}(\mathbf{F})$. A quasivariety $\mathcal{Q}$ is almost structurally complete (ASC) provided that every $\mathcal{Q}$-active quasi-identity holds in $\mathcal{Q}$.

Lemma 3.2. Let $\mathcal{Q}$ be an $S C$ quasivariety. Then every nontrivial algebra $\mathbf{B}$ from $\mathcal{Q}$ admits a homomorphism into some elementary extension of $\mathbf{F}$.

Proof. By Proposition 2.1 and the assumption that $\mathcal{Q}$ is SC, the algebra $\mathbf{B}$ belongs to $\mathrm{SPE}_{\mathrm{el}}(\mathbf{F})$. This means that for each pair of two distinct elements $a$ and $b$ from $B$ there is a homomorphism $h: \mathbf{B} \rightarrow \mathbf{G}$, where $\mathbf{G} \in \mathrm{E}_{\mathrm{el}}(\mathbf{F})$, separating $a$ and $b$. Since $\mathbf{B}$ is nontrivial, there exists at least one such homomorphism.

Theorem 3.3. Let $\mathcal{Q}$ be an ASC quasivariety. Then the following conditions are equivalent

(1) $\mathcal{Q}$ is $S C$;

(2) every nontrivial algebra from $\mathcal{Q}$ admits a homomorphism into some elementary extension of $\mathbf{F}$; 
(3) every nontrivial $\mathcal{Q}$-finitely presented algebra admits a homomorphism into F.

Proof.

$(1) \Rightarrow(2)$ It follows from Lemma 3.2.

$(2) \Rightarrow(3)$ It follows from Fact 2.3 Point (2).

$(3) \Rightarrow(1)$ Let $q=(\forall \bar{x})[\varphi(\bar{x}) \rightarrow \psi(\bar{x})]$ be a $\mathcal{Q}$-admissible quasi-identity. We want to show that $\mathcal{Q} \models q$. Since $\mathcal{Q}$ is ASC, we may assume that $q$ is $\mathcal{Q}$-passive. This exactly means that $\mathbf{P}_{\varphi(\bar{x})}$ does not admit a homomorphism into $\mathbf{F}$. Then Condition (3) says that $\mathbf{P}_{\varphi(\bar{x})}$ is trivial. Hence, by Fact 2.3 point $(1), \mathcal{Q} \models q$ (whatever $\psi$ is).

Corollary 3.4. If $\mathcal{Q}$ is an $A S C$ quasivariety such that $\mathbf{F}$ has an elementary extension with an idempotent element, then $\mathcal{Q}$ is $S C$.

\section{Semisimple QUASIVARIETIES}

Let us now prove a lemma which is built upon [1, Corollary 2.8].

Lemma 4.1. Let $\mathcal{Q}$ be an $S C$ quasivariety such that no elementary extension of $\mathbf{F}$ has an idempotent element. If $\mathbf{S}$ is a $\mathcal{Q}$-simple algebra and $\mathbf{A}$ is a nontrivial algebra from $\mathcal{Q}$, then $\mathbf{S}$ embeds into some elementary extension of $\mathbf{A}$.

Proof. By Lemma 3.2, the lack of idempotent elements in every elementary extension of $\mathbf{F}$ yields the lack of idempotent elements in every nontrivial algebra $\mathbf{B} \in \mathcal{Q}$. Thus, by the $\mathcal{Q}$-simplicity of $\mathbf{S}$ every homomorphism from $\mathbf{S}$ into such $\mathbf{B}$ is injective. It follows that we only need to verify the existence of any homomorphism from $\mathbf{S}$ into some elementary extension of $\mathbf{A}$. This will be accomplished by showing that

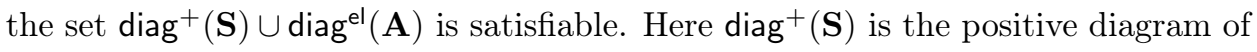
$\mathbf{S}$, i.e., the set of atomic sentences which are valid in the expansion of $\mathbf{S}$ obtained by adding constants corresponding to all elements of $S$. And diag ${ }^{\text {el }}(\mathbf{A})$ is the elementary diagram of $\mathbf{A}$, i.e., the set of first order sentences which are valid in the analogous expansion of $\mathbf{A}[10,18]$.

By the compactness theorem, it is enough to show that for every finite $\Sigma \subseteq$ $\operatorname{diag}^{+}(\mathbf{S})$ the set $\Sigma \cup \operatorname{diag}^{\mathrm{el}}(\mathbf{A})$ is satisfiable. Let $\sigma(\bar{x})$ be the formula obtained from the conjunction $\Lambda \Sigma$ by replacing every constant which is not a constant of $\mathbf{S}$ with a new variable. Then the satisfiability of $\Sigma \cup \operatorname{diag}^{\mathrm{el}}(\mathbf{A})$ is equivalent to the satisfiability of $\{\sigma(\bar{x})\} \cup \operatorname{diag}^{\text {el }}(\mathbf{A})$. The latter will be shown by the verification that $\mathbf{A} \models(\exists \bar{x}) \sigma(\bar{x})$.

By Lemma 3.2, there is a homomorphism from $\mathbf{S}$ into some elementary extension $\mathbf{G}$ of $\mathbf{F}$. Since $\sigma(\bar{x})$ is positive and $\mathbf{S} \models(\exists \bar{x}) \sigma(\bar{x})$, we have $\mathbf{G} \models(\exists \bar{x}) \sigma(\bar{x})$ and also $\mathbf{F} \models(\exists \bar{x}) \sigma(\bar{x})$. By the freeness of $\mathbf{F}$ there also exists a homomorphism from $\mathbf{F}$ into A. Thus, using once more the positivity of $\sigma(\bar{x})$ we obtain $\mathbf{A} \models(\exists \bar{x}) \sigma(\bar{x})$.

At this point we are able to characterize SC quasivarieties among semisimple ASC quasivarieties.

Theorem 4.2. Let $\mathcal{Q}$ be an $A S C$ semisimple quasivariety. Then $\mathcal{Q}$ is $S C$ if and only if there exists an elementary extension of $\mathbf{F}$ with an idempotent element or $\mathcal{Q}$ is minimal. 
Proof. If there exists an elementary extension of $\mathbf{F}$ with an idempotent element, Corollary 3.4 implies that $\mathcal{Q}$ is SC. If $\mathcal{Q}$ is minimal, then it has exactly two subquasivarieties: the trivial one and itself. One of them is $Q(\mathbf{F})$ and it cannot be the trivial one since $\mathbf{F}$ is nontrivial. Hence $\mathcal{Q}$ is also $\mathrm{SC}$.

For the converse implication assume that $\mathcal{Q}$ is an SC quasivariety such that elementary extensions of $\mathbf{F}$ do not have idempotent elements. Let $\mathbf{A}$ be any nontrivial algebra from $\mathcal{Q}$. Lemma 4.1 implies that all $\mathcal{Q}$-simple algebras are in $Q(\mathbf{A})$. Thus the semisimplicity and Proposition 2.2 yield that $\mathcal{Q}=\mathrm{Q}(\mathbf{A})$. This shows that $\mathcal{Q}$ is minimal.

When an additional finiteness condition is imposed the formulation of Theorem 4.2 may be simplified. Note however that Example 5.10 shows that such simplification is not possible in general.

Corollary 4.3. Let $\mathcal{Q}$ be an ASC semisimple quasivariety. Assume that either the language of $\mathcal{Q}$ is finite or $\mathbf{F}$ has a finite subalgebra. Then $\mathcal{Q}$ is $S C$ if and only if $\mathbf{F}$ has an idempotent element or $\mathcal{Q}$ is minimal.

Proof. By Theorem 4.2, it is enough to show that if there exists an elementary extension $\mathbf{G}$ of $\mathbf{F}$ with an idempotent element, then $\mathbf{F}$ also has an idempotent element.

Assume first that the language of $\mathcal{Q}$ is finite. Then the existence of an idempotent element is expressible by a first order sentence. Since $\mathbf{G}$ and $\mathbf{F}$ satisfy the same first order sentences, $\mathbf{F}$ has an idempotent element.

Now assume that $\mathbf{A}$ is a subalgebra of $\mathbf{F}$ with finite carrier. By [8, Theorem V.2.16], G elementarily embeds into some ultrapower $\mathbf{F}^{I} / U$. Thus there is an idempotent element $e$ in $\mathbf{F}^{I} / U$. Let $h: \mathbf{F} \rightarrow \mathbf{A}$ be any homomorphism. Its existence is guaranteed by the freeness of $\mathbf{F}$. We have also a homomorphism $h^{*}: \mathbf{F}^{I} / U \rightarrow$ $\mathbf{A}^{I} / U$ given by $t / U \mapsto h(t) / U$. Then $h^{*}(e)$ is idempotent in $\mathbf{A}^{I} / U$. But the finiteness of $\mathbf{A}$ yields that $\mathbf{A}$ and $\mathbf{A}^{I} / U$ are isomorphic [8, Theorem IV.6.5]. Hence $\mathbf{A}$ and $\mathbf{F}$ have an idempotent element.

\section{Discriminator VARIETIES}

The ternary operation on a set given by

$$
\operatorname{disc}(a, b, c)= \begin{cases}a & \text { if } a \neq b \\ c & \text { if } a=b\end{cases}
$$

is called the discriminator operation. A variety $\mathcal{V}$ is a discriminator variety if there exists a class $\mathcal{K}$ generating $\mathcal{V}$ and a term whose interpretation is the discriminator operation in every member of $\mathcal{K}$. The following facts reveal why the results from Section 4 are applicable for discriminator varieties.

Proposition 5.1. Let $\mathcal{V}$ be a discriminator variety generated by a class $\mathcal{K}$. Assume that there is a term whose interpretation is the discriminator operation in every member of $\mathcal{K}$. Then

(1) $\mathcal{V}$ is $A S C$; 
(2) $\mathcal{V}$ is semisimple;

(3) A nontrivial algebra from $\mathcal{V}$ is simple if and only if it belongs to $\mathrm{SP}_{\mathrm{U}}(\mathcal{K})$.

Proof. For (1) note that $\mathcal{V}$ has projective unification [7, Theorem 3.1] and this yields ASC [13, Corollary 5.1]. For (2) and (3) see [8, Theorem IV.9.4] or [6, Lemma 1.3].

A relevant fact about discriminator varieties is that if they are minimal (as varieties) then they are also minimal as quasivarieties. As far as we know the general case is a new result. However, this was already proved in [5] under some fairly general additional hypothesis. It includes the cases when the language is finite or the variety is locally finite. Note also that in the locally finite case even congruencemodularity yields that minimal varieties are minimal as quasivarieties [3, Corollary 13]. Actually, the following general fact may be deduced from [21, Theorem 3.1]: a locally finite minimal variety is minimal as a quasivariety iff it possesses, up to isomorphism, only one subdirectly irreducible algebra. In [3, Examples 14 and 15] the reader may find examples of minimal varieties which are not minimal as quasivarieties. One of them is locally finite and another is an arithmetical variety.

In the proof we make use of Boolean products, and some of their properties. Let us recall here that Boolean products are subdirect products with some additional requirements. For the precise definition and the proof of the fundamental theorem below we refer the reader to e.g. [8, Theorem 9.4].

Theorem 5.2. Every algebra in a discriminator variety is isomorphic with a Boolean product of trivial and simple factors.

We shall also need the following preservation result.

Lemma 5.3 ([9, Lemma 9.3]). Boolean products preserve the satisfaction of primitive positive sentences, i.e., sentences of the form $\left.(\exists \bar{x})\left[\bigwedge p_{i}(\bar{x})\right) \approx q_{i}(\bar{x})\right]$. This means that if such a sentence is true in every factor then it is true in the Boolean product.

Proposition 5.4. If $\mathcal{V}$ is a minimal discriminator variety, then $\mathcal{V}$ is a minimal quasivariety.

Proof. Let $q$ be a quasi-identity and suppose that there is a nontrivial algebra $\mathbf{A}$ from $\mathcal{V}$ satisfying $q$. Our aim is to show that $\mathcal{V} \models q$. By [32, Theorem 2.4], we have that there are terms $s, t, u, v$ such that

$$
\mathcal{V} \mid=q \leftrightarrow[(\forall \bar{x}) s(\bar{x}) \approx t(\bar{x}) \vee(\forall \bar{x}) u(\bar{x}) \not \approx v(\bar{x})] .
$$

Since $\mathcal{V}$ is a minimal variety, either

$$
\mathcal{V} \vDash(\forall \bar{x}) s(\bar{x}) \approx t(\bar{x})
$$

and then clearly $\mathcal{V} \models q$, or the subvariety of $\mathcal{V}$ defined by $(\forall \bar{x}) s(\bar{x}) \approx t(\bar{x})$ is trivial. Thus in the latter case we have

$$
\mathbf{A} \models(\forall \bar{x}) u(\bar{x}) \not v v(\bar{x}) .
$$


In view of Theorem 5.2, we can suppose that $\mathbf{A} \leqslant \prod\left\{\mathbf{A}_{i}: i \in I\right\}$ is a Boolean product, where each $\mathbf{A}_{i}$ is simple or trivial. Now Lemma 5.3 yields that there exists $i \in I$ such that

$$
\mathbf{A}_{i} \models(\forall \bar{x}) u(\bar{x}) \not \approx v(\bar{x}) .
$$

Note that $\mathbf{A}_{i}$ is nontrivial and hence $\mathbf{A}_{i}$ is simple. Since $\mathcal{V}$ is a minimal variety, it is generated by $\mathbf{A}_{i}$. Thus by (3) of Proposition 5.1, we have

$$
\mathcal{V}_{S} \subseteq \operatorname{SP}_{\mathrm{U}}\left(\mathbf{A}_{i}\right),
$$

where $\mathcal{V}_{S}$ denotes the class of simple algebras in $\mathcal{V}$. Hence

$$
\mathcal{V}_{S} \models(\forall \bar{x}) u(\bar{x}) \not \approx v(\bar{x})
$$

since the satisfaction of $(\forall \bar{x}) r(\bar{x}) \not \approx s(\bar{x})$ is preserved by $\mathrm{S}$ and $\mathrm{Pu}$ class operators. It is also preserved by forming nontrivial subdirect products. Thus Proposition 2.2 implies that

$$
\mathcal{V}-\{\text { trivial algebras }\} \models(\forall \bar{x}) u(\bar{x}) \not \nsim v(\bar{x}) .
$$

This finally gives that $\mathcal{V}=q$.

Theorem 5.5. Let $\mathcal{V}$ be a discriminator variety. Then $\mathcal{V}$ is $S C$ if and only if there exists an elementary extension of $\mathbf{F}$ with an idempotent element or $\mathcal{V}$ is a minimal (as a variety or quasivariety).

Proof. It follows from Theorem 4.2, points (1) and (2) of Proposition 5.1 and Proposition 5.4.

The following corollary shows the advantage of dealing with ASC instead of SC. It is a quite common situation in algebraic logic when there is no idempotent element in any extension of $\mathbf{F}$.

Corollary 5.6. Let $\mathcal{V}$ be a discriminator variety. Assume that there are two distinct constants in $\mathbf{F}$. Then $\mathcal{V}$ is $S C$ if and only if it is minimal.

Proof. Indeed, an idempotent element, by the definition, is a one-element subalgebra. But the smallest subalgebra of any extension of $\mathbf{F}$ has at least two elements.

Example 5.7. Let us consider the variety of two-dimensional (representable) cylindric algebras $(\mathcal{R}) \mathcal{C A}_{2}$ [17]. It is known that it is a discriminator variety with continuum many subvarieties [4, Theorem 4.2]. But it has only two minimal subvarieties [4, Corollary 7.5]. Thus, by Corollary 5.6 and Proposition 5.1 Point (1), there are continuum many subvarieties of $\mathcal{V}$ which are ASC but are not SC.

Example 5.8. We have a similar situation for relation algebras. All (Representable) relation algebras form a discriminator variety. It has exactly three minimal subvarieties [31]. There exists continuum many varieties of (representable) relation algebras [20, Theorem 7.8]. All of them are ASC.

By imposing the same finiteness condition as in Corollary 4.3 we again obtain a certain simplification. 
Corollary 5.9. Let $\mathcal{V}$ be a discriminator variety. Assume that either the language of $\mathcal{V}$ is finite or $\mathbf{F}$ has a finite subalgebra. Then $\mathcal{V}$ is $S C$ if and only if $\mathbf{F}$ has an idempotent element or $\mathcal{V}$ is a minimal variety.

Example 5.10. We will construct an $\mathrm{SC}$ discriminator variety $\mathcal{V}$ which is not minimal (as a variety and hence also as a quasivariety) and its free algebras have no idempotent element.

Let $\mathcal{W}$ be the variety in the language with denumerable many unary operations $f_{i}, i \in \mathbb{N}$, defined by the identities

$$
\begin{gathered}
(\forall x) f_{i}\left(f_{j}(x)\right) \approx f_{j}\left(f_{i}(x)\right) \\
(\forall x) f_{i}\left(f_{i}(x)\right) \approx f_{i}(x)
\end{gathered}
$$

for all $i, j \in \mathbb{N}$. Let $\mathbf{A}$ be the algebra in $\mathcal{W}$ with the carrier $A=\{a, b\}$ and such that $f_{i}(a)=b$ for all $i \in \mathbb{N}$. Let $\mathbf{B}$ be a free algebra for $\mathcal{W}$ of rank at least 1 . Let us expand the language of $\mathcal{W}$ by adding one ternary function symbol disc. Let $\mathbf{A}_{\text {disc }}$ and $\mathbf{B}_{\text {disc }}$ be the expansions of $\mathbf{A}$ and $\mathbf{B}$ respectively to the new language where disc is interpreted as the discriminator operation. Let $\mathcal{V}$ be the variety generated by $\mathbf{A}_{\text {disc }}$ and $\mathbf{B}_{\text {disc }}$. Then $\mathcal{V}$ is a discriminator variety. Each free algebra for $\mathcal{V}$ does not have any idempotent element since $\mathbf{B}_{\text {disc }}$ does not. Also $\mathcal{V}$ is not minimal. In order to see this note that $\mathbf{B}_{\text {disc }}$ is simple and does not belong to $\mathbf{S}\left(\mathbf{A}_{\text {disc }}\right)$. Since $\mathbf{A}_{\text {disc }}$ is finite, $\mathrm{SP}_{\mathbf{U}}\left(\mathbf{A}_{\text {disc }}\right)=\mathrm{S}\left(\mathbf{A}_{\text {disc }}\right)$. Then Proposition 5.1 Point (3) yields that $\mathbf{B}_{\text {disc }} \notin \mathrm{V}\left(\mathbf{A}_{\text {disc }}\right)$.

But $\mathbf{F}$ has an ultrapower with an idempotent element, and hence $\mathcal{V}$ is SC. To see this let us take an element $u \in F$ and consider a sequence $\bar{u}$ in $F^{\mathbb{N}}$ given by $k \mapsto f_{k-1}\left(f_{k-2}\left(\cdots f_{0}(u) \cdots\right)\right)$. Now if $U$ is a non-principal ultrafilter over $\mathbb{N}$, then $\bar{u} / U$ is idempotent in $\mathbf{F}^{\mathbb{N}} / U$.

Example 5.11. Here we will present a minimal discriminator $\mathcal{V}$ variety with a countable algebra $\mathbf{S}$ which does not admit a homomorphism into $\mathbf{F}$. Recall that by Theorem 5.5, $\mathcal{V}$ is SC. Thus, by Theorem 3.3, every nontrivial $\mathcal{V}$-finitely presented algebra admits a homomorphism into $\mathbf{F}$. The presented variety is in a finite language. Since in such varieties every finite algebra is finitely presented, every finite nontrivial algebra from $\mathcal{V}$ admits a homomorphism into $\mathbf{F}$.

Let $\mathbf{A}=(\mathbb{N}, s$, disc $)$ be the algebra, with the set of natural numbers as its carrier and with the successor and the discriminator operations on $\mathbb{N}$ as the basic operations. Let $\mathcal{V}=\mathrm{V}(\mathbf{A})$. By Proposition 5.1 point (3), every simple algebra in $\mathcal{V}$ has a subalgebra isomorphic to $\mathbf{A}$. Thus $\mathcal{V}$ is minimal and SC. For $\mathbf{S}$ we take a simple algebra $(\mathbb{Z}, s$, disc) defined similarly as $\mathbf{A}$ but with the set of integers as the carrier. Note that there is an embedding of $\mathbf{S}$ into $\mathbf{A}^{\mathbb{N}} / U$, where $U$ is a non-principal ultrafilter on $\mathbb{N}$, given by

$$
k \mapsto(i \mapsto \max (i+k, 0)) / U
$$

And indeed, the algebra $\mathbf{S}$ does not admit a homomorphism into any free algebra for $\mathcal{V}$. In order to see this observe that we have the following fact. 
Claim. Let $\mathbf{G}$ be free for $\mathcal{V}$. Then, the operation s in $\mathbf{G}$ is injective. Moreover, for every $a \in G$, there exist (necessarily unique) $n \in \mathbb{N}$ and $b \in G$ such that $a=s^{n}(b)$ and $b \notin s(G)$.

Proof. Free algebras for $\mathcal{V}$ are isomorphic to subalgebras of powers of $\mathbf{A}$. For instance, $\mathbf{F}(1)$ is isomorphic to the subalgebra of $\mathbf{A}^{A}$ generated by the identity function on $A$. So it is enough to verify the property from the claim for $\mathbf{G}$ which is a subalgebra of $\mathbf{A}^{I}$ for some $I$.

The injectivity of $s$ in $\mathbf{G}$ follows from the injectivity of $s$ in $\mathbf{A}$. Let $n$ be the largest number $k$ such that the function $i \mapsto a(i)-k, i \in I$, belongs to $G$ (note that $k$ is bounded above by $a(0))$. Take $b: i \mapsto a(i)-n$ for $i \in I$.

Suppose that there exists a homomorphism $h: \mathbf{S} \rightarrow \mathbf{G}$, where $\mathbf{G}$ is free for $\mathcal{V}$. Let $b$ and $n$ be as in the claim for $a=h(0)$. The injectivity of $s$ in $\mathbf{G}$ yields that $b=h(-n)$. Then $b=s(h(n-1))$. But this is impossible, since $b \notin s(G)$. This gives a contradiction and we conclude that such $h$ does not exist.

\section{Conclusion}

We solved the SC vs ASC problem in the case of semisimple quasivarieties. In particular, we obtained a characterization of SC discriminator varieties. Our results shows that SC property may be very restrictive compared to ASC. It is a common situation that for a discriminator variety the only SC subvarieties are minimal ones while all of them are ASC. In our opinion, it shows the advantage of dealing with ASC instead of SC.

\section{REFERENCES}

[1] Clifford Bergman. Structural completeness in algebra and logic. In Algebraic logic (Budapest, 1988), volume 54 of Colloq. Math. Soc. János Bolyai, pages 59-73. North-Holland, Amsterdam, 1991.

[2] Clifford Bergman. Universal Algebra: Fundamentals and Selected Topics, volume 301 of Pure and Applied Mathematics (Boca Raton). CRC Press, Boca Raton, FL, 2012.

[3] Clifford Bergman and Ralph McKenzie. Minimal varieties and quasivarieties. J. Austral. Math. Soc. Ser. A, 48(1):133-147, 1990.

[4] Nick Bezhanishvili. Varieties of two-dimensional cylindric algebras. II. Algebra Universalis, 51(2-3):177-206, 2004.

[5] Javier Blanco, Miguel Campercholi, and Diego Vaggione. The subquasivariety lattice of a discriminator variety. Adv. Math., 159(1):18-50, 2001.

[6] S. Bulman-Fleming and H. Werner. Equational compactness in quasi-primal varieties. Algebra Universalis, 7(1):33-46, 1977.

[7] Stanley Burris. Discriminator varieties and symbolic computation. J. Symbolic Comput., 13(2):175-207, 1992.

[8] Stanley Burris and H. P. Sankappanavar. A course in universal algebra, volume 78 of Graduate Texts in Mathematics. Springer-Verlag, New York, 1981. The Millennium Edition is available at http://www.math. uwaterloo.ca/ snburris/htdocs/ualg.html.

[9] Stanley Burris and Heinrich Werner. Sheaf constructions and their elementary properties. Trans. Amer. Math. Soc., 248(2):269-309, 1979.

[10] C. C. Chang and H. J. Keisler. Model theory, volume 73 of Studies in Logic and the Foundations of Mathematics. North-Holland Publishing Co., Amsterdam, third edition, 1990. 
[11] Wojciech Dzik. Transparent unifiers in modal logics with self-conjugate operators. Bull. Sect. Logic Univ. Eódź, 35(2-3):73-83, 2006.

[12] Wojciech Dzik. Remarks on projective unifiers. Bull. Sect. Logic Univ. Eódż, 40(1-2):37-46, 2011.

[13] Wojciech Dzik and Michał M. Stronkowski. Almost structural completeness; an algebraic approach. arXiv:1402.5495, 2014.

[14] Roberto Giuntini, Hector Freytes, Antonio Ledda, and Francesco Paoli. A discriminator variety of Gödel algebras with operators arising in quantum computation. Fuzzy Sets and Systems, 160(8):1082-1098, 2009.

[15] Viktor A. Gorbunov. Algebraic theory of quasivarieties. Siberian School of Algebra and Logic. Consultants Bureau, New York, 1998. Translated from the Russian.

[16] Paul R. Halmos. Algebraic logic. Chelsea Publishing Co., New York, 1962.

[17] Leon Henkin, J. Donald Monk, and Alfred Tarski. Cylindric algebras. Part I and II, volume 64 of Studies in Logic and the Foundations of Mathematics. North-Holland Publishing Co., Amsterdam, 1985.

[18] Wilfrid Hodges. Model theory, volume 42 of Encyclopedia of Mathematics and its Applications. Cambridge University Press, Cambridge, 1993.

[19] Peter Jipsen. Discriminator varieties of Boolean algebras with residuated operators. In Algebraic methods in logic and in computer science (Warsaw, 1991), volume 28 of Banach Center Publ., pages 239-252. Polish Acad. Sci., Warsaw, 1993.

[20] Bjarni Jónsson. Varieties of relation algebras. Algebra Universalis, 15(3):273-298, 1982.

[21] Keith A. Kearnes and Ágnes Szendrei. A characterization of minimal locally finite varieties. Trans. Amer. Math. Soc., 349(5):1749-1768, 1997.

[22] T. Kowalski and M. Kracht. Semisimple varieties of modal algebras. Studia Logica, 83(13):351-363, 2006.

[23] Tomasz Kowalski. Varieties of tense algebras. Rep. Math. Logic, 32:53-95, 1998.

[24] Tomasz Kowalski. Semisimplicity, EDPC and discriminator varieties of residuated lattices. Studia Logica, 77(2):255-265, 2004.

[25] Roger D. Maddux. Relation algebras, volume 150 of Studies in Logic and the Foundations of Mathematics. Elsevier, Amsterdam, 2006.

[26] A. I. Mal'cev. Algebraic systems. Springer-Verlag, New York-Heidelberg, 1973.

[27] George Metcalfe and Christoph Röthlisberger. Admissibility in finitely generated quasivarieties. Log. Methods Comput. Sci., 9(2):2:09, 19, 2013.

[28] V. L. Murskir. The existence of a finite basis of identities, and other properties of "almost all" finite algebras. Problemy Kibernet., 30:43-56, 1975.

[29] Alden F. Pixley. Functionally complete algebras generating distributive and permutable classes. Math. Z., 114:361-372, 1970.

[30] Witold A. Pogorzelski. Structural completeness of the propositional calculus. Bull. Acad. Polon. Sci. Sér. Sci. Math. Astronom. Phys., 19:349-351, 1971.

[31] Alfred Tarski. Equationally complete rings and relation algebras. Proc. Neder. Akad. Wet., 59:39-46, 1956.

[32] Diego Vaggione. Free algebras in discriminator varieties. Algebra Universalis, 34(3):391-403, 1995.

[33] Heinrich Werner. Discriminator-algebras, volume 6 of Studien zur Algebra und ihre Anwendungen [Studies in Algebra and its Applications]. Akademie-Verlag, Berlin, 1978. Algebraic representation and model theoretic properties.

[34] Andrzej Wroński. Transparent unification problem. Rep. Math. Logic, (29):105-107 (1996), 1995. First German-Polish Workshop on Logic \& Logical Philosophy (Bachotek, 1995). 
Facultad de Matemática, Astronomía y Física, Ciudad Universitaria, Córdoba, Argentina

E-mail address: camper@famaf.unc.edu.ar

Faculty of Mathematics and Information Sciences, Warsaw University of TechNOLOGY POLAND

E-mail address: m.stronkowski@mini.pw.edu.pl

Facultad de Matemática, Astronomía y Física, Ciudad Universitaria, Córdoba, Argentina

E-mail address: vaggione@famaf.unc.edu.ar 